\title{
Cálculo dos custos do reprocessamento de pinças de uso único utilizadas em cirurgia video-assistida*
}

\author{
CALCULATION OF THE REPROCESSING COSTS OF SINGLE-USE TONGS \\ USED IN VIDEO-ASSISTED SURGERIES
}

\section{CÁLCULO DE LOS COSTOS DEL REPROCESAMIENTO DE PINZAS DE USO ÚNICO UTILIZADAS EN CIRUGÍA VIDEO-ASISTIDA.}

\author{
Eliane Molina Psaltikidis ${ }^{1}$, Kazuko Uchikawa Graziano² ${ }^{2}$ Fábio Frezatti ${ }^{3}$
}

\begin{abstract}
* Artigo extraído da dissertação "Proposta metodológica para análise dos custos do reprocessamento de pinças de uso único utilizadas em cirurgia videoassistida", Escola de Enfermagem da Universidade de São Paulo (EEUSP), 2004

1 Enfermeira. Mestre pelo Programa de Pós-Graduação em Saúde do Adulto da EEUSP. Gerente de Enfermagem do Hospital Novo Atibaia eliane.enf@ hospitalnovo.com.br

2 Enfermeira. Livre docente. Professora do Departamento de Enfermagem Cirúrgica da EEUSP

3 Administrador. Livre docente. Professor do Departamento de Contabilidade e Atuária da FEA-USP e Coordenador do Programa de PósGraduação em Controladoria e Contabilidade da FEA-USP.
\end{abstract}

\begin{abstract}
RESUMO
O trabalho objetivou desenvolver proposta metodológica para cálculo dos custos do reprocessamento de pinças de cirurgia vídeoassistida de uso único. $\mathrm{O}$ reuso freqüente desses artigos ocorre pelo alto custo, entretanto, poucos estudos foram desenvolvidos a respeito desse enfoque. Elaborou-se um fluxograma com cada fase de reprocessamento, permitindo identificação dos componentes dos custos quanto à mãode-obra, materiais e gastos indiretos. Pautado nesses dados, pôde-se construir a proposta metodológica para o cálculo de custos, baseada no método de custeio por absorção, incluindo a planilha para coleta de dados.
\end{abstract}

\section{DESCRITORES \\ Instrumentos cirúrgicos. \\ Reutilização de equipamento. \\ Custos e análise de custo. \\ Cirurgia vídeo-assistida. \\ Equipamentos descartáveis.}

\section{ABSTRACT}

The frequent reuse of disposable forceps instruments is justified by their high cost. However, few studies have been carried out on this topic. The objective of this study was to develop a methodological proposal for calculating the reprocessing costs for these instruments used in videoassisted surgery. A flowchart was developed for each phase of the reprocessing. This allowed subsequent identification of the cost components in terms of labor, materials and indirect expenses. From these data, a methodological proposal for cost calculation could be created, based on the Full Absorption Costing Method, including the spreadsheet for data collection.

\section{KEY WORDS}

Surgical instruments.

Equipment reuse.

Costs and cost analysis.

Video-assisted surgery.

Disposable equipment.

\section{RESUMEN}

El trabajo tuvo como objetivo desarrollar propuesta metodológica para el cálculo de los costos del reprocesamiento de pinzas de cirugía video-asistida de uso único. El uso frecuente de esos artículos ocurre por el alto costo, no obstante, pocos estudios fueron desarrollados respecto a ese enfoque. Se elaboró un fluxograma con cada fase del reprocesamiento, permitiendo la identificación de los componentes de los costos en cuanto a la mano de obra, materiales y gastos indirectos. Teniendo como pauta esos datos, se puede construir la propuesta metodológica para el cálculo de costos, con base en el método de costeo por absorción, incluyendo la planilla para recolección de datos.

\section{DESCRIPTORES}

Instrumentos quirúrgicos. Equipo reutilizado.

Costos y análisis de costo. Cirugía asistida por vídeo. Equipos desechables. 


\section{INTRODUÇÃO}

O desenvolvimento tecnológico na assistência à saúde tem gerado enorme volume de produtos médico-hospitalares classificados como de uso único (ou descartáveis) que são, em geral, confeccionados com materiais mais baratos e termossensíveis. O ideal seria que apresentassem baixo preço de compra para serem consumidos em larga escala. No entanto, verifica-se, cada vez mais, a produção de artigos de uso único com materiais nobres, para finalidades muito específicas em procedimentos médico-cirúrgicos ${ }^{(1-3)}$.

Isto pode ser observado nas cirurgias vídeo-assistidas que adotam diversos artigos de uso único como pinças de dissecção, pinças de apreensão, instrumentais de corte, trocartes e grampeadores descartáveis. Apesar dos inúmeros benefícios do avanço tecnológico, a elevação dos custos destes procedimentos é alvo de preocupação. Assim, várias instituições de diferentes países têm adotado o reuso desses itens ${ }^{(1)}$.

O reuso de artigos de uso único tem sido normatizado pelo Ministério da Saúde e pela Agência Nacional de Vigilância Sanitária (ANVISA) desde $1985^{(4)}$. Está em vigor a Portaria ${ }^{\circ} 4$ de 1986 que pró́be o reuso de somente 11 tipos de artigos, todos de baixo custo ${ }^{(5)}$. Portanto, qualquer produto médico-hospitalar não incluso nessa lista, poderá ser reprocessado, desde que sejam observadas as condições de risco que oferece, isto é, assegurando-se de que não transmita agentes infecciosos, não exponha o paciente a resíduos tóxicos e que a sua função não esteja alterada ${ }^{(1)}$. Assim, verifica-se que portaria não atende mais às necessidades de definição do que é possível ou não reusar, principalmente em razão dos novos artigos que, constantemente, são lançados no mercado, a maioria de alta complexidade. Uma nova portaria está sendo desenvolvida pela ANVISA para adaptar a normatização às necessidades atuais ${ }^{(6-8)}$.

O reuso de artigos de uso único é discutido e estudado sob os aspectos técnicos, legais, éticos e de segurança ${ }^{(9-13)}$. No reuso de artigos de laparoscopia, os riscos potenciais ao paciente são: presença de matéria orgânica residual devido à estrutura que dificulta o processo de limpeza, quebra do instrumento pelos usos repetidos, danos à matéria-prima do artigo por produtos e métodos empregados na limpeza e esterilização, articulações endurecidas, perda da qualidade de corte, podendo ocasionar prolongamento do tempo cirúrgico e perfuração de órgãos interno ${ }^{(11)}$. A garantia de qualidade do artigo de reuso deveria ser comparável à oferecida pelo fabricante para o primeiro uso quanto à apirogenicidade, funcionalidade, esterilidade e atoxicidade ${ }^{(9)}$.

Embora a análise do custo do reuso devesse ser a primeira etapa do processo decisório sobre o reuso de qualquer artigo de uso único, a complexidade e a carência dos métodos específicos para conhecimento dos custos do reproces- samento de artigos de uso único são dificuldades enfrentadas pelos que se dispõem a realizar esta análise.

O estudo sobre custos é uma área relativamente nova para os enfermeiros e seus principais conceitos devem ficar claros. Custos são os gastos relativos a bens ou serviços utilizados na produção de outros bens e serviços. Podem ser classificados em: diretos (podem ser diretamente apropriados aos produtos, bastando que haja uma medida de consumo) e indiretos (não oferecem condição de medida objetiva e qualquer tentativa de alocação é feita de maneira estimada, muitas vezes, arbitrária). Podem ainda ser classificados em: fixos (mantêm-se constantes, independente do volume de produtos elaborados ou de serviços prestados) e variáveis (dependem diretamente do volume de produção ou de serviços prestados) $)^{(14)}$.

Custeio por absorção é o método que consiste na apropriação de todos os custos de produção aos bens elaborados; todos os gastos relativos ao esforço de fabricação são distribuídos para todos os produtos feitos ${ }^{(14)}$. Neste caso, todos os custos (diretos e indiretos) são alocados para cada produto gerado. É a metodologia mais usada, sendo adotada pela contabilidade físcal para cálculo do imposto de renda e também na contabilidade gerencial das entidades. Para este método, o grande desafio consiste em identificar critérios adequados para alocação dos custos indiretos, permitindo que sejam distribuídos nos produtos, sem gerar distorções ${ }^{(14-15)}$.

A análise de custos do reuso envolve, pelo menos, três variáveis: mão-de-obra, materiais e gastos gerais (também denominados custos indiretos de fabricação/prestação de serviços). Os custos diretos da mão-de-obra e de materiais podem ser medidos facilmente e contêm os processos de limpeza, inspeção, identificação, embalagem e esterilização do artigo. Os custos indiretos tais como os gastos referentes à depreciação e manutenção de equipamentos, infra-estrutura, monitorização, controle de qualidade, treinamento e reciclagem periódica da equipe que efetua o reprocessamento são mais difíceis de calcular, pois envolvem alocação ${ }^{(10-11,15)}$.

Devido a importância do controle de custos nas instituições hospitalares, têm sido, cada vez mais freqüente, que administradores solicitem dos profissionais de saúde subsídios técnicos confiáveis quanto à existência ou não de vantagem econômica no reuso dos artigos de uso único. No entanto, a formação e atuação do profissional de saúde salienta o paradigma de que "a saúde não tem preço". Isto o leva a sentir aversão a questões relacionadas a custos, mantendo-o em uma posição romântica ou cômoda frente aos fortes interesses econômicos que determinam as ações de saúde, sejam em macro ou microestruturas. A consciência de que a saúde não tem preço, mas tem custos e de que os recursos para a saúde, sejam públicos ou privados, não são inesgotáveis, obriga as instituições e seus profissionais a fazerem revisão de suas práticas ${ }^{(16-17)}$. 
Frente a isto, o presente estudo teve como objetivos, relativamente ao reprocessamento de pinças de dissecção, apreensão e corte de uso único empregados em cirurgias vídeo-assistidas:

- Identificar e descrever os passos envolvidos;

- Desenvolver uma proposta metodológica para cálculo dos custos do respectivo reprocessamento.

Foram considerados dados para levantamento dos custos que pudessem ser obtidos pelos enfermeiros de Central de Materiais e Esterilização (CME), em sua realidade local. Este opção descartou a possibilidade do emprego de métodos mais apurados de alocação de custos pela sua complexidade e pelo dispêndio de forças para sua aplicação, na prática do profissional enfermeiro de CME.

\section{MÉTODO}

Neste estudo, foi adotada a pesquisa metodológica que, refere-se às investigações de métodos de obtenção, organização e análise de dados, tratando da elaboração, validação e avaliação dos instrumentos e técnicas de pes$q u i s a^{(18)}$. A meta deste tipo de pesquisa é a elaboração de um instrumento confiável que possa ser utilizado posteriormente por outros pesquisadores.

O desenvolvimento da proposta metodológica para análise de custos usou como base teórica os fundamentos da contabilidade de custos, especificamente o método de cus- teio por absorção. Os artigos de uso único investigados restringiram-se às pinças de dissecção, apreensão e corte em razão de apresentarem estruturas semelhantes, serem utilizadas na grande maioria das cirurgias vídeo-assistidas e serem freqüentemente reprocessadas pelas instituições hospitalares, por causa do alto custo.

Inicialmente, foi elaborado um fluxograma com os passos do reprocessamento tendo-se listado os componentes dos custos do reprocessamento dos artigos em estudo, com base na literatura e na experiência da autora. Estes documentos foram avaliados por três enfermeiros atuantes em Central de Material e Esterilização que realizam reprocessamento desses artigos, com objetivo de detectar etapas ou componentes de custo que estivessem ausentes.

Em seguida, foi elaborado o instrumento para o cálculo dos custos do reprocessamento dos artigos. Esta etapa foi desenvolvida com auxílio de pesquisadores da área de controladoria e contabilidade, nos assuntos técnicos referentes à contabilidade de custos. A proposta metodológica também foi submetida à apreciação de dois especialistas em reuso de artigos de uso único.

\section{RESULTADOS}

O fluxograma de reprocessamento, destacou cada etapa necessária para possibilitar o reuso das pinças de uso único empregadas em cirurgia vídeo-assistidas, conforme demonstra a figura 1 .

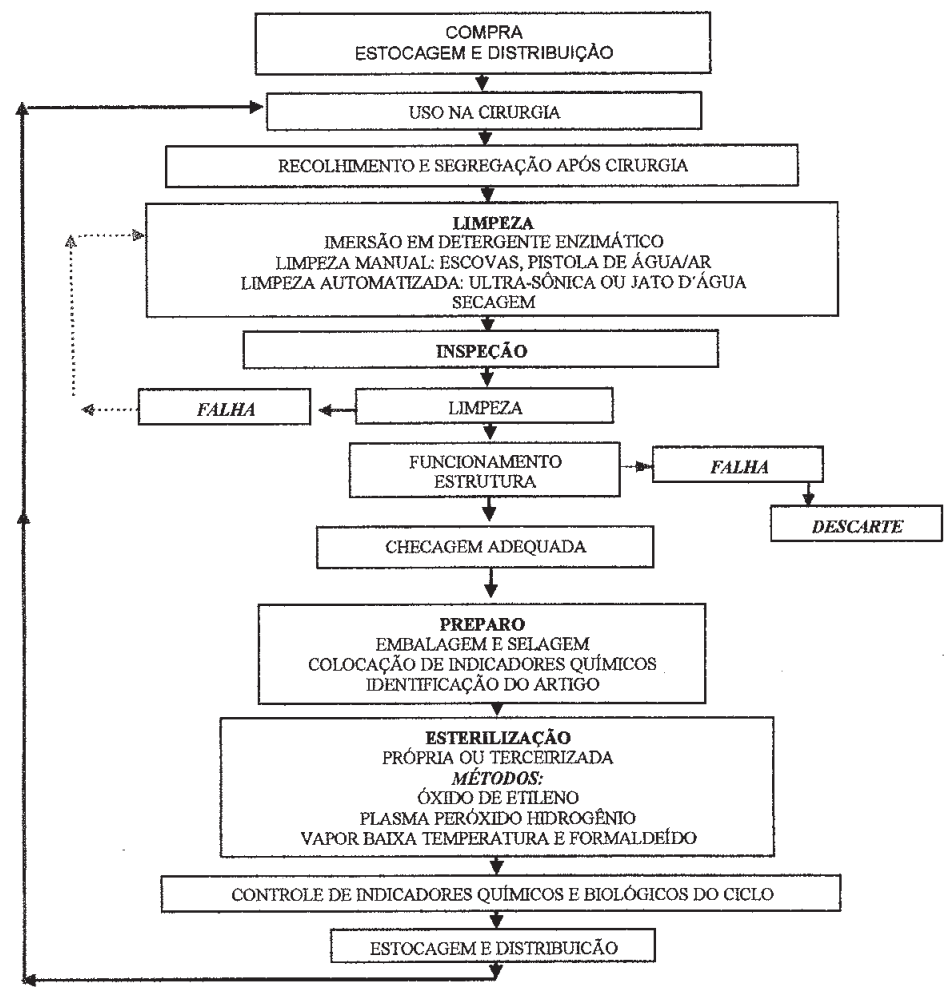

Figura 1 - Fluxograma de reprocessamento de pinças de apreensão, dissecção e de corte de uso único, utilizadas em cirurgia vídeo-assistida. 
Todos os artigos de uso único passam pelas etapas de compra, estocagem, distribuição e uso. Quando há a rotina do reuso, estes artigos, ao invés de serem descartados, são recolhidos e segregados para início do processo de limpeza manual e automatizada.

A fase da secagem pode ser efetuada com campos limpos, ar comprimido ou ação gravitacional. Após essa etapa, a inspeção do artigo é realizada quanto a: limpeza, funcionamento e integridade da estrutura. Caso haja permanência de sujidade, o artigo deve retornar ao início do processo de limpeza, até que se apresente em condições de continuar no reprocessamento. $\mathrm{O}$ funcionamento do artigo deve ser verificado quanto ao alinhamento das lâminas das tesouras, à apreensão das pinças, às travas e ao acionamento das articulações. Se houver falha, o artigo deve ser desprezado. Também devem ser desprezados artigos com falhas na estrutura, tais como: rachaduras, rasgos na capa protetora das pinças ou quebra de algum componente.

A fase de preparo inclui a embalagem e identificação dos artigos e a colocação de indicadores químicos, específicos ao tipo de esterilização adotada. Após, inicia-se a fase de esterilização, própria ou terceirizada. O controle de qualidade do reprocessamento deve garantir a efetividade do processo de esterilização, permitindo que o artigo seja estocado e distribuído para um próximo uso.

Foi descartada a possibilidade do artigo ser submetido à desinfecção de alto nível por ser método inadequado aos artigos em estudo, pois são críticos, ou seja, entram em contato com tecidos e órgãos estéreis e leito vascular. Também não foi contemplada a possibilidade de esterilização por solução germicida, por considerar-se que o processo não é o mais indicado, pelo risco de recontaminação e pela dificuldade de enxágüe adequado, oferecendo risco ao paciente.

A listagem dos componentes de custos do reprocessamento foi elaborada com base no fluxograma, e foram considerados: o tempo da execução de cada etapa pelos profissionais, o emprego de equipamentos (lavadoras, esterilizadoras, seladoras, pistolas de água/ar) e o emprego dos diversos insumos (detergente, lubrificante, embalagens, etiquetas). Também foram apreciados os gastos com controle de qualidade do reprocessamento, tais como: indicadores químicos e biológicos, controle microbiológico dos artigos reprocessados e as análises de pirógenos e de permanência de resíduos do processo de esterilização.

A planilha de cálculo de custos do reprocessamento foi desenvolvida (Anexo 1) com base no fluxograma do reprocessamento e na listagem dos componentes do custo. A metodologia para definição dos custos de cada componente das etapas do reprocessamento foi estabelecida com acompanhamento dos pesquisadores da área de controladoria e contabilidade. Os critérios para alocação foram estabelecidos com base na experiência dos autores sobre o funcionamento das CME, tal como no custo de energia elétrica, estabelecido em $10 \%$ do consumo total da instituição devido à grande quantidade de equipamentos elétricos de alta demanda exigidos para o reprocessamento dos materiais médico-hospitalares.

A planilha de coleta de dados (Anexo 2) foi elaborada com base na planilha de cálculos de custos. A coleta de dados deve ser referente a três meses consecutivos visando obter resultados representativos que permitam as projeções anuais de reprocessamento. Para os dados medidos em minutos ou segundos, foram propostas seis tomadas de tempo, utilizando-se cronômetro. Alguns dados tais como: número de pinças descartadas e sistema de controle do número de reusos, mesmo não tendo correlação direta com o cálculo de custos, foram considerados para melhor entendimento do reprocessamento.

As planilhas e a metodologia propostas foram avaliadas por especialistas em reuso de artigos de uso único: Dra. Evelinda Trindade (larga experiência em avaliação de tecnologias e economia em saúde no Canadá e desenvolvimento de projetos de Tecnovigilância e Hospitais Sentinelas da ANVISA) e Dra. Cristina Toscano (especialista em epidemiologia pelo Centro de Controle Prevenção de Doenças dos Estados Unidos da América e consultora internacional da Organização Pan-Americana da Saúde, tendo coordenado investigações de surtos de infecções hospitalares em diversos países).

A proposta metodológica construída foi aplicada em três hospitais do Estado de São Paulo, para que fosse validada. Nesta aplicação, os resultados obtidos demonstraram que a proposta metodológica mostrou-se apropriada para cálculo e análise de custos do reprocessamento das pinças escolhidas, permitindo identificação dos custos diretos e indiretos, fixos e variáveis, em cada caso analisado.

Como demonstrativo, no Anexo 1, constam os custos obtidos no Hospital Caso 1. Este caso foi escolhido por ser o único dos pesquisados que efetua todo o reprocessamento sem participação de serviço tercerizado. Consiste em uma Instituição privada, de atendimento especializado, com 210 leitos, localizada na cidade de São Paulo. O período analisado foi o trimestre de maio a julho de 2003, quando foram realizadas 1.587 cirurgias, sendo $388(24,45 \%)$ por videoassistidas. No Hospital Caso 1, o custo do reprocessamento foi de $\mathrm{R} \$ 9,374$. Os custos fixos contribuiram com $59,21 \%$ do custo total, os variáveis foram decorrentes com os gastos com embalagens $(40,78 \%)$. O custo do reprocessamento praticado correspondeu a apenas $0,99 \%$ do valor do artigo, tomando por base a pinça de corte que possui o maior preço de compra. 


\section{CONCLUSÃO}

O presente trabalho permite concluir que:

1. O reprocessamento das pinças de dissecção, apreensão e corte utilizadas em cirurgia video-assistida é um trabalho minucioso, com diversas etapas seqüenciais: recolhimento e segregação, limpeza (manual e automatizada), secagem, inspeção (da limpeza, do funcionamento e da estrutura física), preparo (embalagem e rotulagem), esterilização, controle de qualidade, estocagem e distribuição.

2. A análise do fluxograma do reprocessamento dos artigos em estudo permitiu a identificação e listagem dos componentes de custos do reprocessamento, em cada etapa, quanto à mão-de-obra, materiais e gastos indiretos, permitindo a construção da metodologia para o cálculo de custos com base no método de custeio por absorção.

\section{REFERÊNCIAS}

(1) Trindade E. Reuso de materiais descartáveis: situação atual e perspectivas. [Apresentado ao $1^{\circ}$ Encontro de Gerentes de Risco da Rede Sentinela; 2002 nov. 27-29; Rio de Janeiro].

(2) Greene VW. Reuse of disposable devices. In: Mayhall CG. Hospital epidemiology and infection control. Baltimore: Williams \& Wilkins; 1996. p. 946-54.

(3) Pinto TJA, Graziano KU. Reprocessamento de artigos médico-hospitalares de uso único. In: Fernandes AT. Infecção hospitalar e suas interfaces na área da saúde. São Paulo: Atheneu, 2000. p. 1070-7.

(4) Brasil. Ministério da Saúde. Reunião de peritos para normalização do uso e reutilização de materiais médico-hospitalares descartáveis no país. Brasília: Centro de Documentação do Ministério da Saúde; 1985.

(5) Brasil. Ministério da Saúde. Portaria n. 4, de 7 de fevereiro de 1986. Diário Oficial da República Federativa do Brasil, Brasília, 12 fev. 1986. Seção 1, p. 2327.

(6) Romero LC. Aspectos legais do reprocessamento de artigos médico-hospitalares de uso único no Brasil. [Apresentado ao $2^{\circ}$ Simpósio Internacional sobre Tendências em Processos de Esterilização para Instituições de Saúde; 1999 fev. 25-27; São Paulo].

(7) Mesiano, RAB. A legislação brasileira: o que acontece em nosso país. [Apresentado ao $4^{\circ}$ Simpósio Internacional "Tendências em Processos de Esterilização para Instituições de Saúde"; 2003 fev. 17-20; São Paulo].

(8) Brasil. Agência Nacional de Vigilância Sanitária. Consulta Pública n. 98, de 6 de dezembro de 2001. [online]. São Paulo: Federação das Santas Casas de Misericórdia, Hospitais e Entidades Filantrópicas do Estado de São Paulo; [s. d.]. Disponível em: <http://www.fesehf.org.br/circulareshtml2001/circular_ 282.htm.> [Acesso em 04 jan. 2004].
A viabilidade do reprocessamento e a análise do custo obtido no Hospital Caso 1 não são objetivo principal deste trabalho, sendo que o foco consiste no desenvolvimento da proposta metodológica, permitindo que as instituições obtenham o custo do reprocessamento em sua realidade. O presente estudo poderá contribuir para a discussão do reuso de artigos de uso único no aspecto que gerou esta prática, ou seja, verificar se há ou não redução de custos por meio desta prática. Também permitirá a descrição do reprocessamento destes artigos, na prática de cada instituição onde for aplicada, possibilitando a deteç̧ão de pontos falhos ou passíveis de melhoria no processo de controle de qualidade.

A metodologia proposta poderá ser adaptada para outros artigos de uso único, subsidiando a tomada de decisão para cada produto. A estrutura da metodologia poderá basear o desenvolvimento de planilha eletrônica que gere os custos do reprocessamento, ao serem digitados os valores de salários e insumos, os tempos de trabalho das etapas específicos de cada instituição.

(9) United States of America. Department of Health and Human Services. Food and Drug Administration. Reprocessing and reuse of single-use devices: review prioritization scheme. Draft Guidance. [online]. Rockville; 2000. Available from: http:// www.fda.gov/cdrh/reuse/1156.pdf [Access at 31 jul. 2001].

(10) Canadian Healthcare Association. The reuse of single-use medicals devices: guidelines for healthcare facilities. Otawa: CHA; 1996. p. 48.

(11) Emergency Care Research Institute (ECRI). Special report: reuse of single-use medical devices: making informed decisions. Plymouth Meeting, USA: Butler Pike; 1996. p. 37-8, 41-7.

(12) Association of PeriOperative Registered Nurses (AORN). Clinical Issues Reuse of single-use devices. AORN J. 2001; May.

(13) Hogan JM, Colonna TE. Products liability implications of reprocessing d reuse of single-use medical devices. Food and Drug Law J. 1998;53:385-402.

(14) Martins E. Contabilidade de custos. São Paulo: Atlas; 2003.

(15) Frezatti F. Orçamento empresarial: planejamento e controle gerencial. São Paulo: Atlas; 1999. Desenvolvimento do plano de negócios; p. 77-161.

(16) Francisco IMF, Castilho V. A enfermagem e o gerenciamento de custos. Rev Esc Enferm USP. 2002;36(2):240-4.

(17) Francisco IMF, Castilho V. O ensino de custos nas escolas de graduação em enfermagem. Rev Esc Enferm USP. 2004;38:(4)317-25.

(18)Polit D, Hungler BP. Fundamentos de pesquisa em enfermagem. $3^{\text {a }}$ ed. Porto Alegre: Artes Médicas; 1995. Delineamento da pesquisa; p. 107-40. 


\section{ANEXO 1}

Planilha de cálculo dos custos do reprocessamento de pinças de apreensão, dissecção e corte de uso único, utilizadas em cirurgia vídeo-assistida. Hospital Caso nº 1 - Maio-Julho - 2003

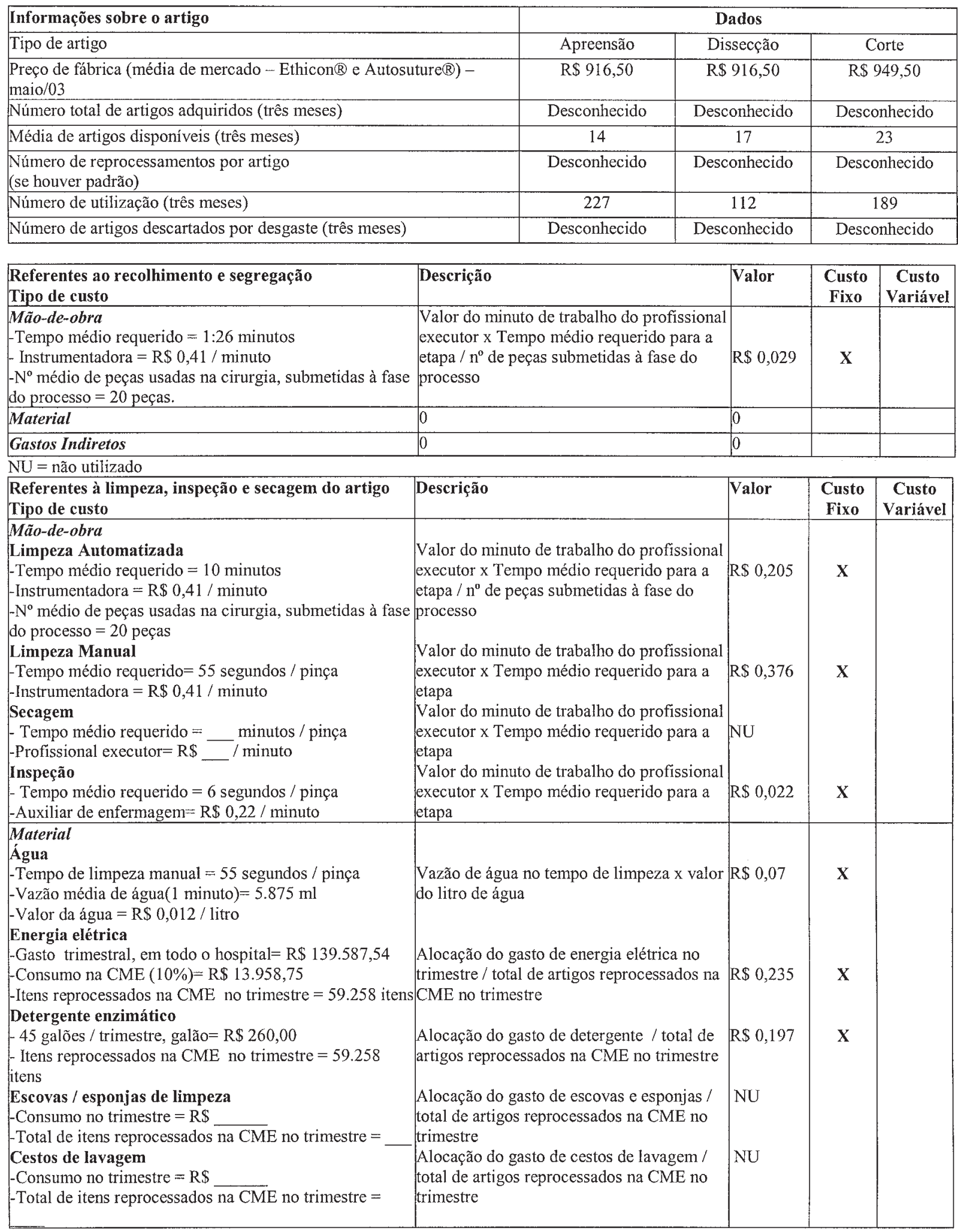

continua... 
(continuação)

\begin{tabular}{|c|c|c|c|c|}
\hline $\begin{array}{l}\text { Campos de secagem } \\
\text { - Consumo no trimestre }=\mathrm{R} \$ \\
\text { - Total de itens reprocessados na CME no trimestre }= \\
\\
\text { Lubrificante } \\
\text { - Consumo no trimestre }=\mathrm{R} \$ \\
\text {-Total de itens reprocessados na CME no trimestre }=\end{array}$ & $\begin{array}{l}\text { Alocação de gasto de campos para secagem } \\
\text { total de artigos reprocessados na CME no } \\
\text { trimestre } \\
\text { Alocação do gasto de lubrificante / total de } \\
\text { artigos reprocessados na CME no trimestre }\end{array}$ & $\mathrm{NU}$ & & \\
\hline $\begin{array}{l}\text { Gastos Indiretos } \\
\text { Lavadora Ultrassônica Unique USC-LDM } \\
\text {-Aquisição por } \mathrm{R} \$ 7.700,00(\mathrm{dez} / 2002), \\
\text {-Depreciação no trimestre }=\mathrm{R} \$ 384,99 \\
\text { - Itens reprocessados na CME no trimestre }=59.258 \text { itens } \\
\text { Pistola de água / ar } \\
\text {-Tipo da pistola }= \\
\text { Depreciação mensal = R } \$ \\
\text { Ar Comprimido } \\
\text { - Custo de manutenção na CME R } \$ 600,00 / \text { trimestre } \\
\text { - Itens reprocessados na CME no trimestre }=59.258 \text { itens } \\
\end{array}$ & $\begin{array}{l}\text { Depreciação da lavadora / total de artigos } \\
\text { reprocessados na CME no trimestre } \\
\text { Depreciação da pistola / total de artigos } \\
\text { reprocessados na CME no trimestre } \\
\text { Alocação do gasto de ar comprimido para } \\
\text { secagem / total de artigos reprocessados na } \\
\text { CME no trimestre }\end{array}$ & NU & $\mathbf{X}$ & \\
\hline \begin{tabular}{|l|} 
Referentes à embalagem do artigo \\
Tipo de custo \\
\end{tabular} & Descrição & Valor & $\begin{array}{c}\text { Custo } \\
\text { Fixo }\end{array}$ & \begin{tabular}{|c|} 
Custo \\
Variável
\end{tabular} \\
\hline $\begin{array}{l}\text { Mão-de-obra } \\
\text {-Tempo médio requerido }=59 \text { segundos } \\
\text {-Auxiliar de enfermagem }=\mathrm{R} \$ 0,22 / \text { minuto }\end{array}$ & $\begin{array}{l}\text { Valor do minuto de trabalho do profissional } \\
\text { executor x Tempo médio requerido para a } \\
\text { etapa }\end{array}$ & $\mathrm{R} \$ 0,216$ & $\mathbf{X}$ & \\
\hline $\begin{array}{l}\text { Material } \\
\text { Tipo da embalagem } \\
\text {-Embalagem Tyvec-rolo } 200 \mathrm{~mm} \times 70 \text { metros = R } \$ 354,00 \\
\text { - Manta de não tecido de } 101 \mathrm{~cm}=\mathrm{R} \$ 1,65 \\
\text { - Manta de não tecido de } 76 \mathrm{~cm}=\mathrm{R} \$ 1,21\end{array}$ & $\begin{array}{l}\text { Uso de } 60 \mathrm{~cm} / \text { pinça } \\
2 \text { mantas de } 101 \mathrm{~cm} \text { para embalagem } \\
\text { externa } / 9 \text { pinças } \\
1 \text { mantas de } 76 \mathrm{~cm} \text { para embalagem interna } \\
9 \text { pinças }\end{array}$ & $\begin{array}{l}\mathrm{R} \$ 3,034 \\
\mathrm{R} \$ 0,366 \\
\mathrm{R} \$ 0,134\end{array}$ & & $\begin{array}{l}\mathbf{X} \\
\mathbf{X} \\
\mathbf{X}\end{array}$ \\
\hline
\end{tabular}

$\mathrm{NU}=$ não utilizado

\begin{tabular}{|c|c|c|c|c|}
\hline $\begin{array}{l}\text { Referentes à embalagem do artigo (cont.) } \\
\text { Tipo de custo }\end{array}$ & Descrição & Valor & $\begin{array}{l}\text { Custo } \\
\text { Fixo }\end{array}$ & $\begin{array}{c}\text { Custo } \\
\text { Variável }\end{array}$ \\
\hline $\begin{array}{l}\text { Indicador químico de esterilização } \\
\text { - Fita adesiva reagente }=\mathrm{R} \$ 59,83 / \text { rolo } 55 \text { metros } \\
\text { - Tira reagente }=\mathrm{R} \$ 105,00 / \text { caixa com } 250 \text { unidades }\end{array}$ & $\begin{array}{l}\text { Uso de } 2 \text { metros } / 9 \text { pinças } \\
\text { Uso de } 1 \text { tira } / 9 \text { pinças }\end{array}$ & $\begin{array}{l}\mathrm{R} \$ 0,242 \\
\mathrm{R} \$ 0,047\end{array}$ & & $\begin{array}{l}\mathbf{X} \\
\mathbf{X}\end{array}$ \\
\hline Etiqueta de identificação do conteúdo & Uso / pinça & $\mathrm{NU}$ & & \\
\hline \begin{tabular}{|l|} 
Gastos Indiretos \\
Seladora \\
Seladora consignada
\end{tabular} & $\begin{array}{l}\text { Depreciação da seladora / total de artigos } \\
\text { reprocessados na CME no trimestre }\end{array}$ & 0 & & \\
\hline $\begin{array}{l}\text { Referentes à esterilização do artigo por serviço } \\
\text { terceirizado } \\
\text { Tipo de custo }\end{array}$ & Descrição & Valor & $\begin{array}{l}\text { Custo } \\
\text { Fixo }\end{array}$ & $\begin{array}{c}\text { Custo } \\
\text { Variável }\end{array}$ \\
\hline $\begin{array}{l}\text { Mão-de-obra } \\
\text {-Tempo requerido para preparo dos artigos e envio para } \\
\text { empresa terceirizada }=\quad \text { minutos } \\
\text { - Profissional executor }=\mathrm{R} \$ \text { \& } \$ \text { minuto } \\
-\mathrm{N}^{\circ} \text { médio de itens enviados }=\ldots \text { itens }\end{array}$ & $\begin{array}{l}\text { Valor do minuto de trabalho do profissional } \\
\text { executor } \mathrm{x} \text { Tempo requerido para preparo e } \\
\text { envio } / \mathrm{n}^{\mathbf{0}} \text { médio de itens }\end{array}$ & NU & & \\
\hline $\begin{array}{l}\text { Material } \\
\text { Preço da esterilização por artigo }\end{array}$ & 0 & NU & & \\
\hline Gastos Indiretos & 0 & 0 & & \\
\hline $\begin{array}{l}\text { Referentes à esterilização do artigo pela própria } \\
\text { instituição } \\
\text { Tipo de custo }\end{array}$ & Descrição & Valor & $\begin{array}{l}\text { Custo } \\
\text { Fixo }\end{array}$ & $\begin{array}{c}\text { Custo } \\
\text { Variável }\end{array}$ \\
\hline $\begin{array}{l}\text { Mão-de-obra } \\
\text { Montagem da carga e listagem dos itens } \\
\text {-Tempo médio requerido }=4: 31 \text { minutos } \\
\text { - Auxiliar de enfermagem }=\mathrm{R} \$ 0,22 / \text { minuto } \\
-\mathrm{N}^{\circ} \text { médio de artigos esterilizados por ciclo }=27 \text { itens } \\
\text { Desmontagem da carga e colocação da etiqueta de lote } \\
\text {-Tempo médio requerido }=9: 23 \text { minutos } \\
- \text { Auxiliar de enfermagem }=\mathrm{R} \$ 0,22 / \text { minuto } \\
-\mathrm{N}^{\circ} \text { médio de artigos esterilizados por ciclo }=27 \text { itens }\end{array}$ & $\begin{array}{l}\text { Valor do minuto de trabalho do profissional } \\
\text { executor } \mathrm{x} \text { Tempo médio requerido para } \\
\text { montagem das cargas } / \mathrm{n}^{\circ} \text { médio de artigos } \\
\text { esterilizados por ciclo } \\
\text { Valor do minuto de trabalho do profissional } \\
\text { executor } \mathrm{x} \text { Tempo médio requerido para } \\
\text { desmontagem das cargas } / \mathrm{n}^{\circ} \text { médio de } \\
\text { artigos esterilizados por ciclo }\end{array}$ & $\begin{array}{l}R \$ 0,036 \\
R \$ 0,076\end{array}$ & $\mathrm{X}$ & \\
\hline
\end{tabular}


(continuação)

\begin{tabular}{|c|c|c|c|c|}
\hline $\begin{array}{l}\text { Acompanhamento do ciclo } \\
\text {-Tempo de ciclo }=50 \text { minutos } \\
\text {-Auxiliar de enfermagem }=\text { RS } 0,22 / \text { minuto } \\
-N^{\circ} \text { médio de artigos esterilizados por ciclo }=27 \text { itens }\end{array}$ & $\begin{array}{l}\text { Valor do minuto de trabalho do profissional } \\
\text { executor x Tempo requerido para } \\
\text { acompanhamento do ciclo de esterilização / } \\
n^{\circ} \text { médio de artigos esterilizados por ciclo }\end{array}$ & $\mathrm{R} \$ 0,407$ & $\mathbf{X}$ & \\
\hline $\begin{array}{l}\text { Material } \\
\text { Agente esterilizante } \\
\text {-Plasma de peróxido de hidrogênio (PPH) } \\
\text { Caixa com } 15 \text { cassetes = R } \$ 3.555,00 \\
\text { Etiqueta de registro do lote de esterilização } \\
\text { Rolo com } 1000 \text { etiquetas }=\mathrm{R} \$ 3,34 \\
\end{array}$ & $\begin{array}{l}\text { Cada cassete possibilita cinco ciclos, } \\
\text { portanto R } \$ 47,40 \text { / ciclo com média de } 27 \\
\text { itens } \\
\text { Uso de } 1 \text { etiqueta / } 9 \text { pinças }\end{array}$ & $\begin{array}{l}\mathrm{R} \$ 1,755 \\
\mathrm{R} \$ 0,0004\end{array}$ & $\mathbf{X}$ & \\
\hline \begin{tabular}{|l|} 
Gastos Indiretos \\
Esterilizador \\
-Autoclave a PPH (Sterrad 100S-ASP Johnson \& Johnson) \\
Aquisição por R $\$ 240.000,00($ set/2000) \\
Depreciação no trimestre $=\mathrm{R} \$ 12.000,00$ \\
-Itens reprocessados por PPH no trimestre = 9.142 itens \\
\end{tabular} & $\begin{array}{l}\text { Depreciação do esterilizador no trimestre / } \\
\text { Total de itens reprocessados por PPH no } \\
\text { trimestre }\end{array}$ & $\mathrm{R} \$ 1,312$ & $\mathbf{X}$ & \\
\hline \begin{tabular}{|l|} 
Referentes à estocagem e dispensação do artigo \\
Tipo de custo
\end{tabular} & Descrição & Valor & $\begin{array}{l}\text { Custo } \\
\text { Fixo }\end{array}$ & $\begin{array}{c}\text { Custo } \\
\text { Variável } \\
\end{array}$ \\
\hline $\begin{array}{l}\text { Mão-de-obra } \\
\text { Estocagem } \\
\text {-Tempo estimado }=30 \text { segundos } / \text { pinça } \\
\text {-Auxiliar de enfermagem }=\mathrm{R} \$ 0,22 / \text { minuto } \\
\text { Controle de Estoque } \\
\text {-Tempo estimado }=60 \text { horas } / \text { trimestre(por item }=4 \text { seg.) } \\
\text { - Itens reprocessados na CME no trimestre }=59.258 \text { itens } \\
\text {-Auxiliar de enfermagem }=\mathrm{RS} 0,22 / \text { minuto } \\
\text { Dispensação } \\
\text { Tempo estimado }=30 \mathrm{segundos} / \text { pinça } \\
\text {-Auxiliar de enfermagem }=\mathrm{R} \$ 0,22 / \text { minuto }\end{array}$ & $\begin{array}{l}\text { Valor do minuto de trabalho do profissional } \\
\text { executor x Tempo estimado para estocagem } \\
\text { Valor do minuto de trabalho do profissional } \\
\text { executor } x \text { Tempo estimado para controle } \\
\text { do estoque } \\
\text { Valor do minuto de trabalho do profissional } \\
\text { executor } x \text { Tempo estimado para } \\
\text { dispensação }\end{array}$ & $\begin{array}{l}\mathrm{R} \$ 0,11 \\
\mathrm{R} \$ 0,015 \\
\mathrm{R} \$ 0,11\end{array}$ & $\mathbf{X}$ & \\
\hline Material & 0 & o & & \\
\hline
\end{tabular}

$\mathrm{NU}=$ não utilizado

Referentes à estocagem e dispensação do artigo (cont.) Tipo de custo

\section{Gastos Indiretos}

Mão-de-obra

Manutenção das condições ambientais

- Tempo estimado $=60$ minutos $/$ trimestre

- Auxiliar de manutenção $=\mathrm{R} \$ 0,11 /$ minuto

-Itens reprocessados na CME no trimestre $=59.258$ itens

-Filtro do ar condicionado = custo desconhecido

Referentes ao controle de qualidade

Tipo de custo

Mão-de-obra

Colocação e retirada de indicadores biológicos (IB)

- Tempo estimado $=4$ horas $/$ trimestre

- Auxiliar de enfermagem $=\mathrm{R} \$ 0,22 /$ minuto

-Itens reprocessados por PPH no trimestre $=9.142$ itens

Preparo e leitura dos indicadores biológicos (IB)

- Tempo estimado $=12$ horas $/$ trimestre

- Enfermeiro $=R \$ 0,39 /$ minuto

- Itens reprocessados por $\mathrm{PPH}$ no trimestre $=9.142$ itens

Material

Indicadores biológicos (IB)

- Indicador biológico específico $=\mathrm{R} \$ 12,18$

Uso semanal de 4 indicadores biológicos, portanto, 48

indicadores / trimestre

- Itens reprocessados por PPH no trimestre $=9.142$ itens

Controle microbiológico

- Tipo de controle microbiológico $=$

- Custo do controle microbiológico $=\mathrm{R} \$$

Análise de pirógenos

-Tipo de análise de pirógenos $=$

- Custo da análise de pirógenos $=\mathrm{R} \$$

Análise de resíduos químicos do agente esterilizante -

cromatografia

-Tipo de análise de resíduos $=$

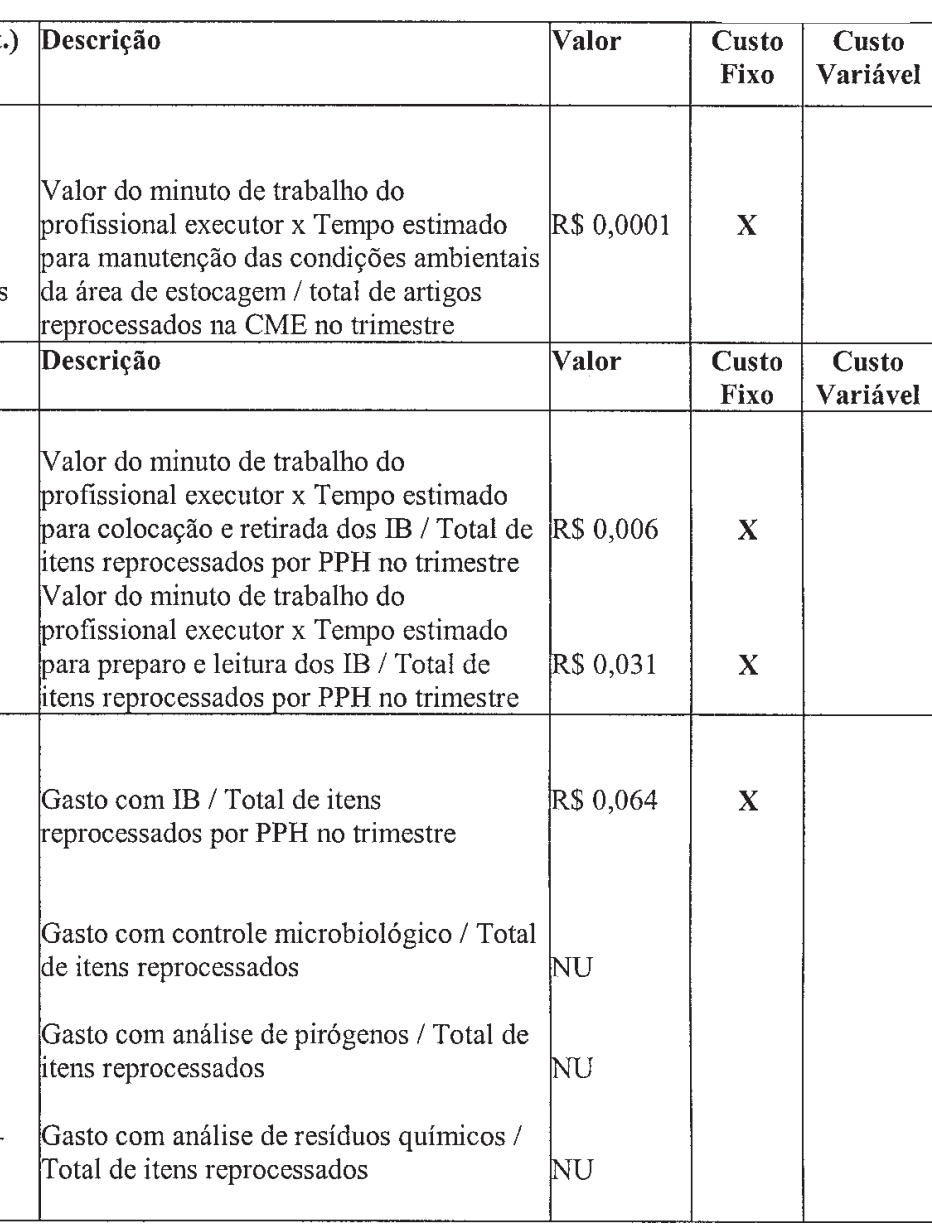

continua... 


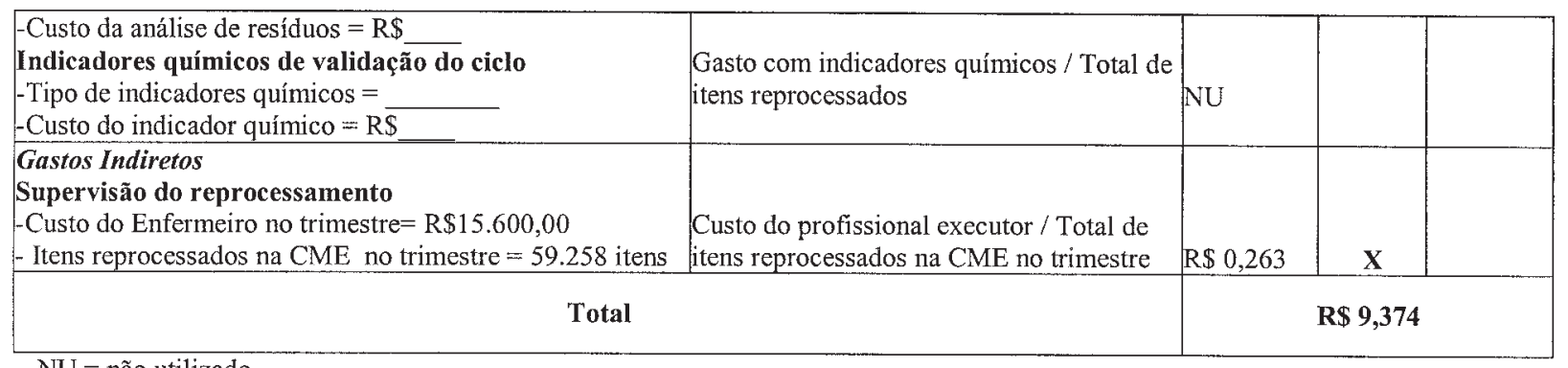

$\mathrm{NU}=$ não utilizado

\section{ANEXO 2}

Planilha de coleta de dados do hospital para cálculo dos custos do reprocessamento de pinças de apreensão, dissecção e corte de uso único, utilizadas em cirurgia vídeo-assistida

Hospital: .................................. Datas de coleta: ........../.... ...................................................

Área Total construída do hospital: __ $\mathrm{m}^{2} \quad \mathrm{~N}^{0}$ de leitos: $\quad$ Área da CME: __ $\mathrm{m}^{2}$

\begin{tabular}{|c|c|c|c|c|c|c|c|c|c|c|}
\hline MATERIAL & FABRICANTE & $\mathrm{AQU}$ & IÇÃOM & NSAL & PINC & $S$ DISPO & IVEIS & PINC & S UTIL & ADAS \\
\hline & CódIGo & MÊs 1 & MÊS 2 & MÊs 3 & MÊs 1 & MÊS 2 & MÊs 3 & MÊS 1 & MÊS 2 & MÊS 3 \\
\hline PINÇA DE APREENSÃO & & & & & & & & & & \\
\hline PINÇA DE DISSECCCÃO & & & & & & & & & & \\
\hline PINÇA DE CORTE & & & & & & & & & & \\
\hline
\end{tabular}

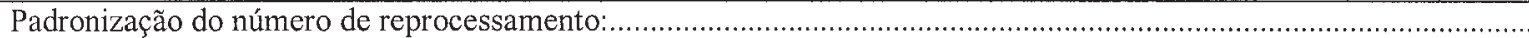

Sistema de controle:.

Recursos humanos da Central de Material e Esterilização:

\begin{tabular}{|l|l|l|l|l|}
\hline Profissionais & \multicolumn{3}{|c|}{ Número por turno } & \multicolumn{1}{|c|}{ Total } \\
\cline { 2 - 5 } Salário bruto, encargos e beneficios & Manhã & Tarde & Noturno & \\
\hline Chefia & & & & \\
\hline Enfermeiros & & & & \\
\hline Técnicos de enfermagem & & & & \\
\hline Auxiliares de enfermagem & & & & \\
\hline Outros & & & & \\
\hline
\end{tabular}

Outros

Cirurgias vídeo-assistidas, segundo tipo de cirurgia e freqüência mensal

\begin{tabular}{|l|c|c|c|}
\hline CIRURGIAS VIDEO-ASSISTIDAS & \multicolumn{3}{|c|}{ NÚMERO MENSAL } \\
\hline & MÊS 1 & \multicolumn{1}{|c|}{ MÊS 3 } \\
\hline & & & \\
\hline & & & \\
\hline & & & \\
\hline Total de cirurgias video-assistidas & & & \\
\hline Total de cirurgias & & & \\
\hline
\end{tabular}

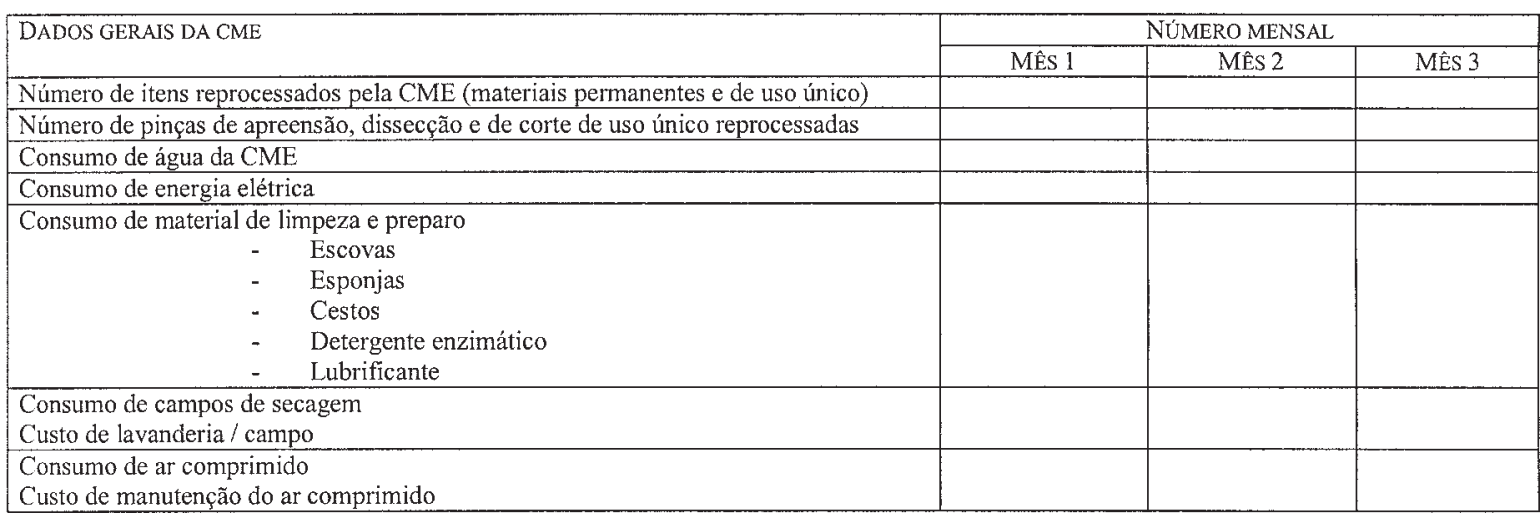


(continuação)

Recolhimento e Segregação

Rotina adotada (distâncias, materiais, executor):

Tomadas de tempo do recolhimento (em minutos e segundos)

\begin{tabular}{|l|l|l|l|l|l|}
\hline Tempo 1 & Tempo 2 & Tempo 3 & Tempo 4 & Tempo 5 & Tempo 6 \\
\hline & & & & & \\
\hline
\end{tabular}

\section{Limpeza, inspeção e secagem}

Rotina adotada (equipamentos, materiais, produtos, executor):

Descrição dos equipamentos para limpeza (fabricante, modelo, capacidade, tempo de ciclo, anos de uso, depreciação, consumo de energia elétrica por ciclo):

Descrição dos detergentes:

Vazão de água pela torneira em 1 minuto (seis tomadas)

\begin{tabular}{|l|l|l|l|l|l|}
\hline Tempo 1 & Tempo 2 & Tempo 3 & Tempo 4 & Tempo 5 & Tempo 6 \\
\hline & & & & & \\
\hline
\end{tabular}

Tomadas de tempo da limpeza (em minutos e segundos)

\begin{tabular}{|c|c|c|c|c|c|}
\hline Tempo 1 & Tempo 2 & Tempo 3 & Tempo 4 & Tempo 5 & Tempo 6 \\
\hline & \multicolumn{3}{|l|}{} & \\
\hline Tomadas de tempo da inspeção (em minutos e segundos) \\
\hline Tempo 1 & Tempo 2 & Tempo 3 & Tempo 4 & Tempo 5 & Tempo 6 \\
\hline
\end{tabular}

Tomadas de tempo da secagem (em minutos e segundos)

\begin{tabular}{|c|c|c|c|c|c|}
\hline Tempo 1 & Tempo 2 & Tempo 3 & Tempo 4 & Tempo 5 & Tempo 6 \\
\hline & & & & & \\
\hline
\end{tabular}

\section{Preparo e embalagem}

Rotina adotada (materiais, executor, selagem, identificação, indicador químico):

Descrição da seladora (fabricante, modelo, anos de uso, consumo de energia elétrica por ciclo):

Descrição do indicador químico (fabricante, produto, consumo por pacote):

Tomadas de tempo do preparo e embalagem (em minutos e segundos)

\begin{tabular}{|c|c|c|c|c|c|}
\hline Tempo 1 & Tempo 2 & Tempo 3 & Tempo 4 & Tempo 5 & Tempo 6 \\
\hline & & & & & \\
\hline
\end{tabular}

\section{Esterilização (terceirizada)}

Rotina adotada (executor, método, insumos, sistema de cobrança, tempo de envio e recebimento):

Tomadas de tempo do preparo e envio do lote (em minutos e segundos)

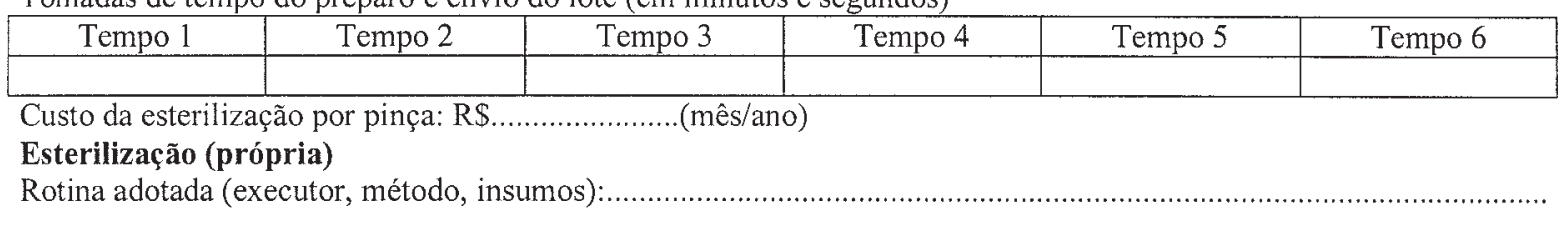

Descrição dos equipamentos esterilizadores (fabricante, modelo, capacidade, tempo de ciclo, anos de uso, depreciação, consumo de energia elétrica por ciclo, insumos):.

Tomadas de tempo da montagem da carga (em minutos e segundos)

\begin{tabular}{l}
\begin{tabular}{|c|c|c|c|c|c|}
\hline Tempo 1 & Tempo 2 & Tempo 3 & Tempo 4 & Tempo 5 & Tempo 6 \\
\hline & & & & & \\
\hline Tomadas de tempo da desmontagem da carga (em minutos e segundos) & Tempo 6 \\
\hline Tempo 1 & Tempo 2 & Tempo 3 & Tempo 4 & Tempo 5 & Tempons \\
\hline
\end{tabular} \\
\hline
\end{tabular}

continua... 
(continuação)

Estocagem e dispensação

Rotina adotada (distâncias, materiais, executor):

Tomadas de tempo da estocagem (em minutos e segundos)

\begin{tabular}{l}
\begin{tabular}{|c|c|c|c|c|c|}
\hline Tempo 1 & Tempo 2 & Tempo 3 & Tempo 4 & Tempo 5 & Tempo 6 \\
\hline & \multicolumn{3}{|l|}{} & & \\
\hline
\end{tabular} \\
Tomadas de tempo do controle do estoque (em minutos e segundos) \\
\hline Tempo 1
\end{tabular}

Tomadas de tempo da dispensação (em minutos e segundos)

\begin{tabular}{|c|c|c|c|c|c|}
\hline Tempo 1 & Tempo 2 & Tempo 3 & Tempo 4 & Tempo 5 & Tempo 6 \\
\hline & & & & & \\
\hline
\end{tabular}

Descrição dos controles ambientais da área do estoque e custos aproximados:

\section{Controle de qualidade}

Rotina adotada (executor, métodos, testes, periodicidade, insumos):

Tomadas de tempo da realização de testes com indicadores químicos e biológicos (em minutos e segundos)

\begin{tabular}{|c|c|c|c|c|c|}
\hline Tempo 1 & Tempo 2 & Tempo 3 & Tempo 4 & Tempo 5 & Tempo 6 \\
\hline & & & & & \\
\hline
\end{tabular}

Tomadas de tempo do encaminhamento e/ou realização de outros testes: controle microbiológico, de pirógenos, de resíduos químicos (em minutos e segundos)

\begin{tabular}{|c|c|c|c|c|c|}
\hline Tempo 1 & Tempo 2 & Tempo 3 & Tempo 4 & Tempo 5 & Tempo 6 \\
\hline & & & & & \\
\hline Tomadas de tempo do controle dos testes de qualidade (em minutos e segundos) \\
\hline Tempo 1 & Tempo 2 & Tempo 3 & Tempo 4 & Tempo 5 & Tempo 6 \\
\hline
\end{tabular}

